\title{
UTILIZACIÓN DEL WHATSAPP Y SUS EFECTOS EN UN MODELO DE LIDERAZGO Y ADMINISTRACIÓN INSTITUCIONAL ${ }^{\star}$
}

\section{USE OF WHATSAPP AND ITS EFFECTS ON A LEADERSHIP AND INSTITUTIONAL ADMINISTRATION MODEL}

Recibido: 15 de marzo de 2019

Evaluado: 4 de abril de 2019

Aprobado: 6 de mayo de 2019

\author{
Luz Carmen Anaya Saladén \\ Universidad EAN \\ Orcid: 0000-0002-7742-1116
}
Sandra Rocío Valbuena Castillo"**
Universidad EAN
Orcid: 0000-0001-7091-5896
Henry Manuel Ortega Jiménez ${ }^{\star \star \star \star}$
Universidad EAN
Orcid: 0000-0002-7963-6233

Naira Yadira Hernández Rojas ${ }^{\star \star \star \star \star}$

Universidad EAN

Orcid: 0000-0001-8665-4803

Cómo citar este artículo:Ortega Jiménez, H. M., Anaya Saladén, L. C., Hernández Rojas, N. Y. y Valbuena Castillo, S. R. (2019). Utilización del WhatsApp y sus efectos en un modelo de liderazgo y administración institucional. Revista Estrategia Organizacional, 8 (2). pp. 49-60. doi: https://doi.org/10.22490/25392786.3431

\footnotetext{
* $\quad$ Artículo de investigación.

** Contadora Pública, Especialista en Administración Financiera, Especialista en Gestión Humana y MBA, Universidad EAN. Correo electrónico: lanaya@universidadean.edu.co

*** Administradora de Empresas, Especialista Gerencia de Mercadeo, Especialista en Gerencia del Servicio, y Máster MBA, Universidad EAN

**** Profesional en Publicidad y Mercadeo, Especialista en Mercadeo y Máster MBA, Universidad EAN.

$\star \star \star \star \star$ Ingeniera de Sistemas y Especialista en Gerencia de Proyectos, Universidad EAN.
} 


\title{
RESUMEN
}

El desarrollo de la presente investigación se basa en el efecto que causa el uso del aplicativo WhatsApp en un contexto laboral. Se estudia específicamente el caso de la Universidad EAN, siendo una herramienta que es utilizada como medio de comunicación entre líderes y colaboradores. La interacción a través del WhatsApp genera efectos tanto positivos como negativos y es interés de esta investigación definir su impacto en el modelo de liderazgo de la Universidad EAN. En el año 2014, esta institución tuvo cambio de dirección y está atravesando un periodo de transformación institucional, mediante esta investigación se definirá el modelo de liderazgo y administración ejercido al interior de la institución y el efecto del aplicativo como medio de comunicación.

Palabras clave: WhatsApp, liderazgo, administración, comunicación, trabajo.

\begin{abstract}
The development of the research is based on the effect it Whatsapp application usage in the workplace context of EAN University, being a tool that is used as a means of communication between leaders and collaborators. The interaction through WhatsApp generates both positive and negative effects and importance of this project is to define its impact on leadership model EAN University. Since the EAN University in 2014 had change of direction and is going through a period of institutional transformation, through this research the model of leadership and administration exercised within the institution and the effect of the application as a communication medium is defined.
\end{abstract}

Keyboards: WhatsApp, Leadership, Administration, Comunication, Work.

\section{INTRODUCCIÓN}

En los últimos años el avance acelerado de la tecnología ha hecho que se transforme el entorno laboral. Más allá de las plataformas que se desarrollan al interior de diversas empresas para la creación de intranet comunicativo, el sistema de mensajería instantánea de WhatsApp, la popular aplicación de chat para teléfonos inteligentes, proyecta la posibilidad de que el teléfono móvil sea una extensión del computador y permita conversaciones y mensajes directos o grupales para difundir actividades propias del ámbito laboral. 
La incursión de esta herramienta de mensajería instantánea a través de teléfonos móviles ha impactado la comunicación entre líderes, pares y equipos de trabajo, de allí la importancia que está investigación le otorga, dado el beneficio que como medio de comunicación puede agregar a las organizaciones.

Creada en el año 2009 por la empresa WhatsApp Inc., (Blog Oficial WhatsApp) y vendida en febrero de 2014 a la empresa Facebook Inc., en la actualidad cuenta con más de 700 millones de usuarios activos (Marín, 2015), lo cual la convierte en una de las aplicaciones más populares del mundo, basando su éxito en la facilidad que tiene todo usuario para registrarse sin necesidad de brindar información y con solo aportar el número de teléfono celular. La popularidad del aplicativo al alcance de todos, ha difundido su uso personal a los ámbitos laborales.

Esta práctica ha invadido el lugar de trabajo, las investigaciones que han tratado temas sobre las aplicaciones de las nuevas tecnologías y sus usos sociales, establecen la alta penetración de las redes sociales personales incluso durante las horas de trabajo, sin embargo, carecen de evidencias que confirmen que el uso de redes sociales virtuales estimule la creatividad o inspire a algunos trabajadores. No obstante, el solo hecho de convertirse en un nuevo medio de comunicación y la rapidez de su funcionalidad le atribuyen características que benefician tanto al trabajador como a la organización.

Por lo expuesto, resulta pertinente la investigación desarrollada en el marco de la Maestría en Administración de Empresas, de la Facultad de Estudios en Ambientes Virtuales de la Universidad EAN, sobre el uso creciente de mensajería en línea para fines laborales y su potencial efecto positivo en el rendimiento del trabajo, el bienestar de las empresas y el liderazgo administrativo.

\section{LIDERAZGO: TRANSFORMACIÓN Y TRANSACCIÓN EN LA TECNOLOGÍA}

El crecimiento de la tecnología que ha transformado la vida cotidiana en los recientes años no sólo ha generado nuevos estilos de vida y que se desarrollen revoluciones estructurales e industriales (Kranzberg y Pursell, 1967; Derry y Williams, 1960), sino que ha logrado la organización de la sociedad y de las unidades productivos (Yoguel, 2008; Alierta, 2011), impactando aspectos como la comunicación entre los equipos de trabajo. En ese contexto, la tecnología de comunicación permite el intercambio de información entre dos individuos y fomenta el desarrollo de la percepción, expectativas y participación que conforman los fundamentos de la comunicación (Drucker, 2011). 
Asimismo, el ámbito laboral es por excelencia el contexto privilegiado para evaluar la posibilidad de interrelacionarse a través de la tecnología y de medir su funcionalidad en la organización laboral, de acuerdo con el esquema que responden las jerarquías y posiciones, líderes y pares, y cómo son respetadas las mismas y sus correspondientes funciones, en el cual la eficiencia y los logros "se intensifican de acuerdo con el grado de satisfacción que se establece en las relaciones laborales. Este grado es directamente proporcional con el de compromiso" (Chadi, 2004, p. 52).

Al respecto, Philip Kotter (citado por Hernández 2013) apunta que "el liderazgo no es más que la actividad o proceso de influenciar a la gente para que se empeñe voluntariamente en el logro de los objetivos del grupo, entendiendo por grupo un sector de la organización con intereses afines". Un liderazgo que se percibe en la influencia entre líderes y seguidores para lograr los objetivos organizacionales por medio del cambio (Lussier, 2010), y en el cual los roles gerenciales de liderazgo (Mintzberg, 2008), incluyen lo interpersonal, lo informativo y las decisiones.

Según la teoría de Bass (1991), bien sea que el liderazgo se defina por atributos de la personalidad, o por las cualidades que surgen en medio de acontecimiento extraordinarios o por las personas que deciden convertirse en líderes aprendiendo las destrezas necesarias, el principio transformador del líder se enfoca en gran medida en su visión, más que en las atribuciones de los seguidores, pues "los lideres transformacionales son conocidos por conmover y cambiar las cosas en gran forma" (Lussier y Achua, 2011).

Por tanto, el liderazgo transformacional es un proceso que cambia individuos, "que tiene en consideración los valores, la ética, los estándares y las metas a largo plazo; que envuelve el conocimiento de las motivaciones de los seguidores, la satisfacción de sus necesidades y el trato como seres humanos completos" (Northouse 2001, citado por Zarate 2010).

Igualmente, dentro del estilo referido como liderazgo transformador, se determina que está enfocado en fortalecer y reconocer el valor de todas las personas. De lo que resulta la necesidad de que las organizaciones sean menos jerárquicas, que el liderazgo transformador coincida con la definición operativa de liderazgo efectivo (Anderson, 1992). Así, el liderazgo transformador incluye visión, planificación, comunicación y acción creativa. Tiene un efecto unificador positivo en un grupo de personas en torno a una serie de valores y creencias claras para cumplir un conjunto claro 
de metas mensurables. El método de transformación impacta simultáneamente en el desarrollo personal y la productividad organizativa de todos los involucrados (Bass 1991).

De acuerdo a la teoría del liderazgo transformador de Bass, los líderes realizan acciones que se basan en indicadores confiables de la satisfacción de los colaboradores y los asociados en una organización. La comunicación efectiva por parte del liderazgo, en primer lugar, ayuda a los empleados y miembros a entender la estrategia global de la organización; en segundo lugar, permite entender cómo pueden contribuir para lograr objetivos claves; y, finalmente, comparten información sobre la situación de la organización y la manera en que la función del colaborador o del miembro está relacionada con los objetivos estratégicos de la organización. Por lo que, los buenos líderes usan la tecnología como una herramienta para alcanzar sus metas. (Bass 1991)

Como resultado de la investigación se evidencia que la Universidad EAN se encuentra en un proceso de transición, en el cual se observan características del modelo de liderazgo transformacional y transaccional, este último siendo el liderazgo que "se distingue por tres características: recompensas condicionadas, gerencia pasiva por excepción y gerencia activa por excepción" (según Burns, 1978; citado por Zárate, 2010).

En ese orden de ideas, en las recompensas condicionadas del liderazgo transaccional, "el comportamiento del subordinado siempre está supeditado a las recompensas". A su vez, la gerencia pasiva por excepción "se refiere al uso de correctivos o castigos por comportamientos no adecuados y por el no logro de metas propuestas". Mientras, la gerencia activa por excepción, "se refiere a descubrir los errores de los subordinados y reforzar la ley y las normas para evitarlos" (Burns 1978).

Finalmente, la integración WhatsApp-liderazgo y los efectos de su relación e impacto en el contexto laboral, merece ser determinado, para ello, se aplicó el método científico como una serie de pasos ordenados que Ilevaran a obtener las respuestas para definir los efectos del uso del WhatsApp en el modelo de liderazgo de la Universidad EAN. 


\section{CORRELACIÓN CUANTITATIVA Y PROCEDIMIENTO METODOLÓGICO}

El carácter correlacional con enfoque cuantitativo experimental de la investigación acerca de los efectos de la utilización del WhatsApp en el modelo de liderazgo de la Universidad EAN, permite tanto describir la relación entre las variables y efectos observados, que como técnica descriptiva indica las coincidencias de respuestas (Salkind, 1999). Para ello, mediante la aplicación de encuestas en la Universidad EAN se busca definir características y grado de asociación de variables. Para determinar los tamaños de la muestra, se definió un segmento de la población que representará a los dos principales grupos de estudio: líderes y colaboradores, la población objeto de estudio fueron 72 líderes, representados por quienes ocupan cargos de coordinadores y directores administrativos, y 76 colaboradores, representados en los cargos de profesionales en cada una de las áreas.

La investigación se diseñó teniendo en cuenta los siguientes parámetros: nivel de confianza del $95 \%$, un margen de error del $5 \%$, porcentaje estimado de la muestra: 50 \%, donde arrojó las siguientes muestras: lideres 59 encuestas y colaboradores 61 encuestas, aplicadas a través de la herramienta Google a la totalidad de las muestras señaladas.

\section{RESULTADOS. LIDERAZGO EN TRANSICIÓN}

Existen diferentes puntos de vista entre colaboradores y líderes respecto al modelo de liderazgo de la Universidad EAN, mientras el $83 \%$ de los colaboradores señalan que el modelo de liderazgo es transaccional, el $100 \%$ de los líderes consideran que es transformacional.

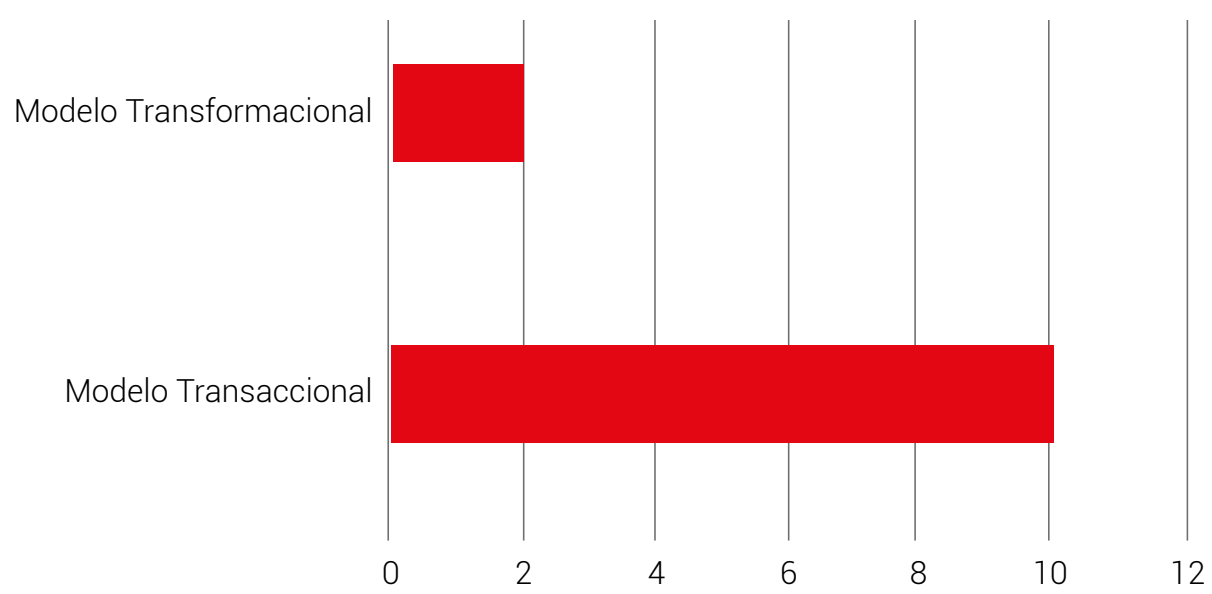

Figura 1. Modelo de liderazgo de la Universidad EAN según los colaboradores.

Fuente: elaboración propia 


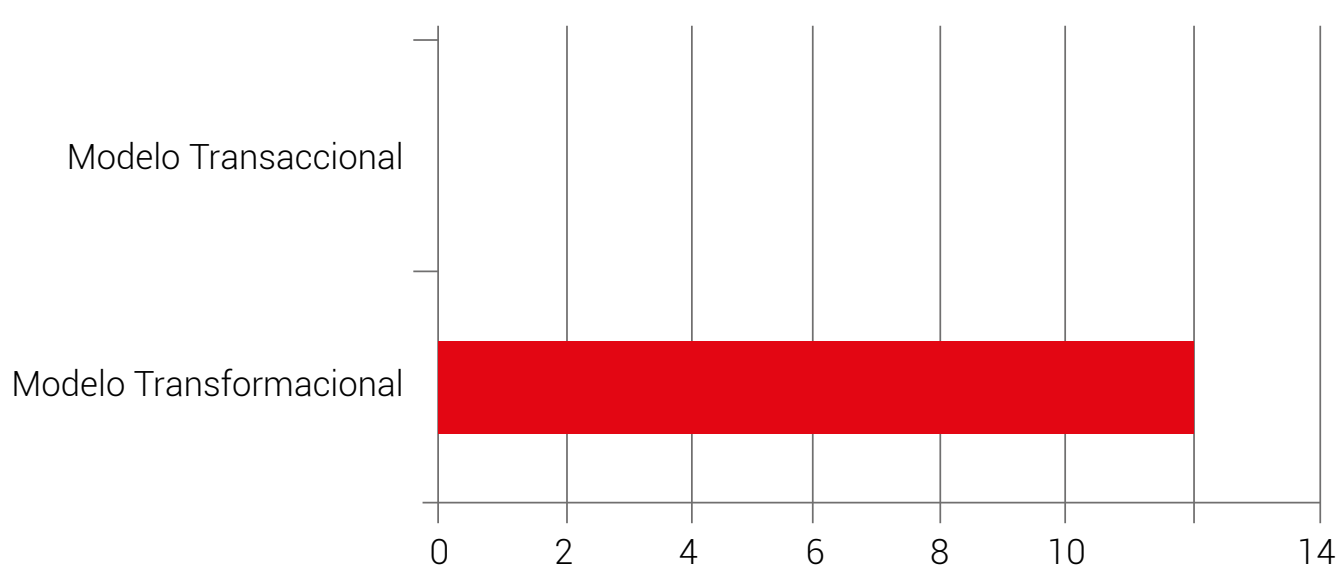

Figura 2. Modelo de liderazgo de la Universidad EAN según los líderes.

Fuente: elaboración propia

Se atribuye este resultado a que la Universidad se encuentra en un momento de transición de la alta gerencia y por lo tanto esta disparidad puede obedecer a la posición que cada líder y colaborador asume frente su concepto de liderazgo en la organización. Este proceso de transición refleja visiones distintas de "colaboradores" y "líderes", sin embargo, requiere que la alta gerencia inicie un trabajo de consolidación del nuevo modelo de liderazgo afianzando los actuales valores.

Como lo muestra el estudio, esta herramienta se ha difundido en la mayoría de colaboradores y líderes de la Universidad EAN, adicionando nuevos métodos al modelo de comunicación en la Institución, que lo benefician con información inmediata, eficaz, rapidez de respuestas, confirmación de recibidos, según indican estos dos grupos analizados.

Dentro de los principales hallazgos, se encontró que el 93\% de líderes y colaboradores usan y tienen instalada la aplicación, los líderes de los procesos de la Universidad EAN utilizan WhatsApp como alternativa en el proceso de comunicación con los colaboradores en un $48 \%$, mientras que el $64 \%$ de colaboradores indica que su jefe inmediato se comunica a través de esta herramienta. 


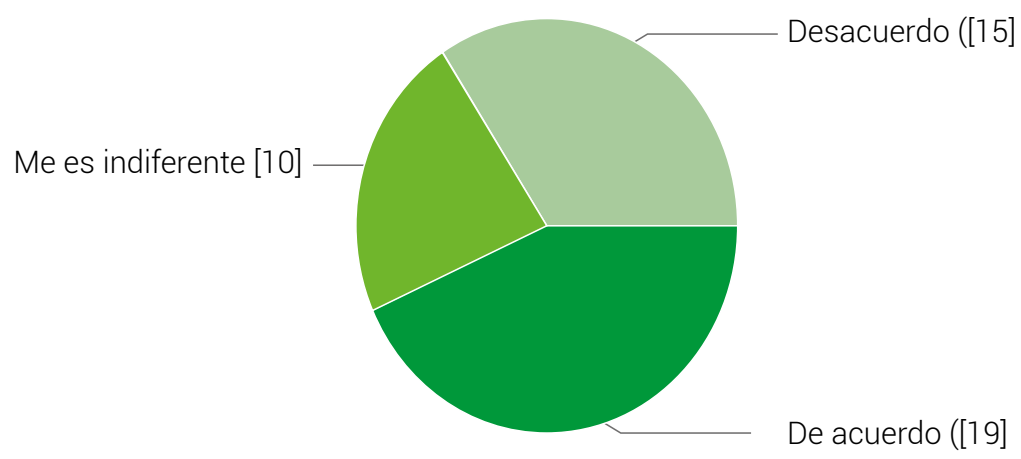

$\begin{array}{lcc} & \text { Cantidad } & \text { Porce } \\ \text { De acuerdo } & 19 & 31.1 \% \\ \text { Me es indiferente } & 10 & 16.4 \% \\ \text { Desacuerdo } & 15 & 24.6 \%\end{array}$

Figura 3. ¿Está de acuerdo o en desacuerdo con que su jefe se comunique por WhatsApp?

Fuente: elaboración propia

La mayoría de los colaboradores (31 \%) que tiene instalada la aplicación WhatsApp está de acuerdo con que su jefe le envíe mensajes a través de esta aplicación y el 16 \% le es indiferente, lo que refleja que esta aplicación es de gran ayuda para la comunicación entre líderes y colaboradores. Un $25 \%$ está en desacuerdo.

Entre los colaboradores que están de acuerdo con el uso de esta aplicación en contexto laboral, el $15 \%$ señala que facilita la comunicación, el $13 \%$ genera inmediatez y el $7 \%$ indica que funciona muy bien al reemplazar llamadas en momentos en que no se puede contestar.

Del 25 \% de colaboradores que está en desacuerdo señala que el uso del celular y la aplicación es netamente personal, e invade el espacio del encuestado. 


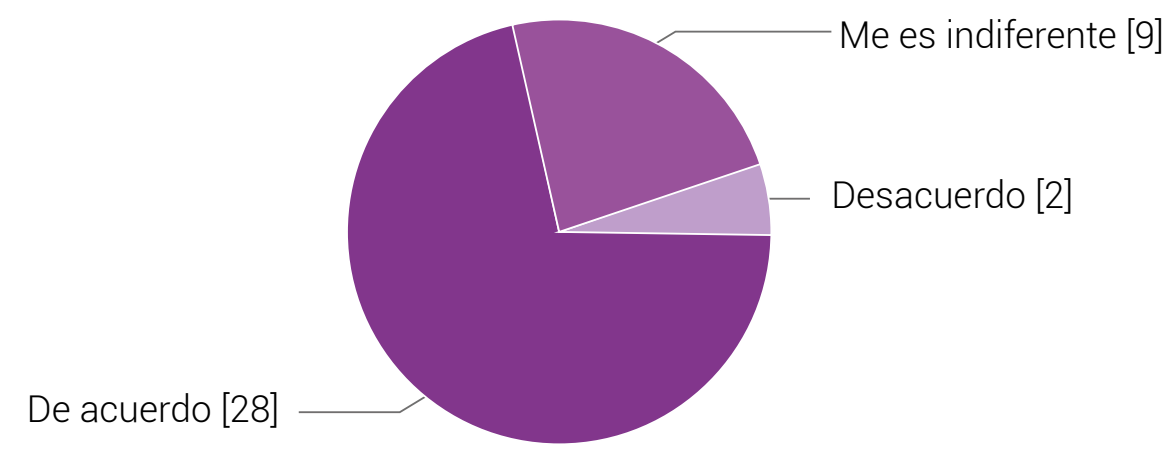

$\begin{array}{lll} & \text { Cantidad } & \text { Porcentaje } \\ \text { De acuerdo } & 28 & 45.9 \% \\ \text { Me es indiferente } & 9 & 14.8 \% \\ \text { Desacuerdo } & 2 & 3.3 \%\end{array}$

Figura 4. Está de acuerdo o en desacuerdo en comunicarse con sus colaboradores, por medio de aplicativos a través del celular.

Fuente: elaboración propia

El $46 \%$ de los líderes indica que está de acuerdo con hacer envíos a través de WhatsApp, el $15 \%$ le es indiferente y tan solo el $3 \%$ está en desacuerdo. La mayoría de los líderes encuestados señalan que están de acuerdo con el uso de la aplicación WhatsApp, ya que facilita la comunicación y genera inmediatez.

Tabla 1. Utilización WhatsApp.

\begin{tabular}{|c|c|c|}
\hline DATOS & COLABORADORES & LÍDERES \\
\hline Tiene instalada la aplicación WhatsApp & $93 \%$ & $93 \%$ \\
\hline De acuerdo y le es indiferente el uso WhatsApp en contexto laboral. & $48 \%$ & $61 \%$ \\
\hline Fortalezas & \multicolumn{2}{|c|}{$\begin{array}{l}\text { - } \text { Genera facilidad } \\
\text { - Inmediatez } \\
\text { - Eficacia } \\
\text { - } \text { Comunicación individual y grupal. }\end{array}$} \\
\hline Debilidades & \multicolumn{2}{|c|}{$\begin{array}{l}\text { - Exige estar conectado } \\
\text { - Exige estar pendiente del WhatsApp } \\
\text { - Genera mayor control } \\
\text { - Invade el aspecto personal }\end{array}$} \\
\hline
\end{tabular}

Fuente: elaboración propia 
Tabla 2. Modelo de liderazgo.

\begin{tabular}{|l|l|l|}
\hline \multicolumn{1}{|c|}{ ENCUESTADOS } & \multicolumn{1}{|c|}{$\begin{array}{c}\text { LIDERAZGO TRANSFORMACIONAL } \\
\text { Bass (1991) }\end{array}$} & $\begin{array}{c}\text { LIDERAZGO TRANSACCIONAL } \\
\text { Burns (1978) }\end{array}$ \\
\hline Colaboradores & $17 \%$ & $83 \%$ \\
\hline Líderes & $100 \%$ & $0 \%$ \\
\hline
\end{tabular}

Fuente: elaboración propia

La investigación ratifica cómo las herramientas de comunicación de la revolución tecnológica son un factor de desarrollo de las empresas y se han convertido en un elemento renovador y de facilidad para la comunicación entre líderes y colaboradores.

\section{CONCLUSIONES}

La utilización del WhatsApp entre los líderes de la Universidad ha facilitado la comunicación relacionada con el dar instrucciones, enviar tareas y establecer contacto directo.

Se evidencia cómo los líderes de la Universidad EAN, independientemente de la generación en que se encuentren, interactúan de una manera directa y más ágil con los colaboradores por medio de la aplicación de mensajería instantánea WhatsApp.

Los líderes que utilizan esta herramienta se comunican de manera informal, desarrollando mejores relaciones humanas con los colaboradores y haciendo interacciones sociales con los diferentes colaboradores.

En la Universidad EAN se evidencia que actualmente no existe un modelo definido de liderazgo, debido a que la institución está viviendo un proceso de transición.

Se observan visiones diferentes en cuanto a lo que se considera liderazgo para "colaboradores" como para "líderes".

El nuevo liderazgo da cabida al uso del WhatsApp y le corresponde al área de Desarrollo Humano definir los mecanismos para que el uso del aplicativo sea un medio más de comunicación del ámbito laboral, sin afectar la vida personal del individuo. 


\section{REFERENCIAS}

Alierta, C. (2011). The Promise of Technology. En S. Dutta, y I. Mia (Eds.), The Global Information Technology Report 2010-2011: Transformations 2.0 (pp. 61-68). Geneva, Switzerland: World Economic Forum Editors.

Anderson. (1992). Transforming Leadership: New Skills for an Extraordinary Future. (n.d.)

Bass, B. (1991) From Transactional to Transformational Leadership: Learning to Share the Vision. (n.d.)

Burns, J. M. (s.f.). "Leadership", Harper and Rowe, New York.

Chadi, M. (2004). Redes sociales en el trabajo social. Buenos Aires: Espacio Editorial.

Derry, T. and Williams, T. (1960). A Short History of Technology: From the Earliest Times to A.D. 1900. New York: Dover Publications.

Drucker, P. (2011). Technology, Management, and Society. Boston: Harvard Business School Publishing.

Hernández, A. (2023). El liderazgo eficaz, factor determinante para el clima organizacional de las empresas. Tesis. Bogotá, Colombia.

Kotter, J. (1998). The Leadership factor. Nueva York: Free Press.

Kranzberg, M. and Pursell, C. (1967). Technology in Western Civilization: Technology in the Twentieth Century. New York: Oxford University Press.

Lussier, R. y Achua, C. (2010). Liderazgo teoría, aplicación y desarrollo de habilidades. México: Cengage Learning.

Lussier, R. y Achua, C., (2011). Liderazgo: Teoría, aplicación y desarrollo de habilidades. Madrid: Cengaje Learning. 
Marín, E. (2015). WhatsApp ya cuenta con más de 700 millones de usuarios activos. Recuperado de: http://hipertextual.com/2015/01/whatsapp-700-millones-usuarios

Mintzberg. (2008). Liderazgo, teoría, aplicación y desarrollo de habilidades. (n.d.).

Morales, O. (s.f.). Estadísticas Twiitter 2014 en Colombia y el mundo. Recuperado de: http://www.oamg. co/blog/social-media/41-estadisticas-twitter-2014-en-colombia-y-el-mundo

Salkind, N. J. (1999). Métodos de investigación. Pearson Educación. Recuperado de: https://books. google.es/books?hl=es\&lr=\&id=3ulW0vVD63wC\&oi=fnd\&pg=PR19\&dq=que+es+la+lnvestigaci\% C3\%B3n+correlacional\&ots=aHHDdb_QaO\&sig=IBaLNPwMfhipFor_KUzmTOmiQUw\#v= onepage $\& \mathrm{q}=$ correlacional $\& \mathrm{f}=\mathrm{false}$

WhatsApp. (s.f.). Blog oficial. Recuperado de: http://blog.whatsapp.com/

Yoguel, G. (2008). Información y conocimiento: las vinculaciones entre difusión de TIC y competencias tecnológicas. En G. Valenti, D. Avaro, y M. Casalet. (Eds.), Instituciones, sociedad del conocimiento y mundo del trabajo. (pp. 295- 326). México: FLACSO. 